\title{
OVERCONVERGENT REAL CLOSED QUANTIFIER ELIMINATION
}

\author{
L. LIPSHITZ AND Z. ROBINSON
}

\begin{abstract}
Let $K$ be the (real closed) field of Puiseux series in $t$ over $\mathbb{R}$ endowed with the natural linear order. Then the elements of the formal power series rings $\mathbb{R} \llbracket \xi_{1}, \ldots, \xi_{n} \rrbracket$ converge $t$-adically on $[-t, t]^{n}$, and hence define functions $[-t, t]^{n} \rightarrow K$. Let $\mathcal{L}$ be the language of ordered fields, enriched with symbols for these functions. By Corollary 3.17, $K$ is o-minimal in $\mathcal{L}$. This result is obtained from a quantifier elimination theorem. The proofs use methods from non-Archimedean analysis.
\end{abstract}

\section{INTRODUCTION}

The main result of this paper is the quantifier elimination theorem, Theorem 2.1, for the real closed field

$$
K:=\bigcup_{n} \mathbb{R}\left(\left(t^{1 / n}\right)\right)
$$

with restricted division and analytic functions given by $t$-adically overconvergent power series with coefficients in $K$. The elimination theorem yields o-minimality results such as the one mentioned in the abstract, Corollary 3.17. We would like to thank Sergei Starchenko for bringing to our attention the question answered in that corollary, which motivated this investigation.

Remark 1.1. Theorem 3.16, together with an example by Hrushovski and Peterzil [7], yields a sentence in an expansion of the language of ordered fields that is false in all expansions of $(\mathbb{R},+, \cdot,<)$, but is true in the real closed field $K$ of Corollary 3.17. This gives a counterexample to a question raised in [5], p. 153, see also [1], p. 3.

Let $K_{1}:=\bigcup_{n} \mathbb{R}\left(\left(t^{1 / n}\right)\right)$ and let $|\cdot|_{t}$ denote the $t$-adic norm on $K_{1}$; i.e., the norm determined by $\left|t^{\alpha}\right|_{t}=2^{-\alpha}, \alpha \in \mathbb{Q}$, and $|c|_{t}=1, c \in \mathbb{R} \backslash\{0\}$. Since $K_{1}$ is a direct union of complete fields, it is a Henselian valued field. Let $<$ denote the linear order on $K_{1}$ determined by the order on $\mathbb{R}$; i.e., $\sum c_{i} t^{i / n}>0$ if, and only if, the leading coefficient is a positive real number. (We will also use $<$ for the order relation on the value group $\left|K_{1}\right|_{t} \subset \mathbb{R}$. This should not cause confusion.) Note that $|\cdot|_{t}$ is the valuation canonically determined by the order $<$ on $K_{1}$ in the sense that the valuation ring coincides with the set of "finite" elements; i.e.,

$$
\left\{a \in K_{1}:|a|_{t} \leq 1\right\}=\left\{a \in K_{1}:-b<a<b \text { for some } b \in \mathbb{R}\right\} .
$$

The residue field $\mathbb{R}$ is a real closed field, the value group $\mathbb{Q}$ is divisible and $K_{1}$ is Henselian, so $K_{1}$ is a real closed field. By $|\cdot|_{<}$denote the quantifier-free definable

2000 Mathematics Subject Classification. Primary 03C64, 32P05, 32B05, 32B20, 03C10, Secondary $03 \mathrm{C} 98,03 \mathrm{C} 60,14 \mathrm{P} 15$.

Supported in part by NSF grant DMS-0401175. 
absolute value $|x|_{<}:=\sqrt{x^{2}}$. Let $K_{2}$ be the completion of $K_{1}$ with respect to $|\cdot|_{t}$, and let $K$ denote either $K_{1}$ or $K_{2}$.

Let

$$
\begin{aligned}
K^{\circ} & :=\left\{x \in K:|x|_{t} \leq 1\right\} \text { and } \\
K^{\circ \circ} & :=\left\{x \in K:|x|_{t}<1\right\} .
\end{aligned}
$$

Let $\xi=\left(\xi_{1}, \ldots, \xi_{n}\right)$ be variables, $\nu=\left(\nu_{1}, \ldots, \nu_{n}\right)$ be indices. By $|\nu|$ denote $\nu_{1}+$ $\cdots+\nu_{n}$. The ring of strictly convergent power series in $\xi$ over $K$ is

$$
K\langle\xi\rangle:=\left\{\sum a_{\nu} \xi^{\nu}: \lim _{|\nu| \rightarrow \infty}\left|a_{\nu}\right|_{t}=0\right\} .
$$

The elements of $K\langle\xi\rangle$ converge on the closed unit polydisc $U=\left(K^{\circ}\right)^{n}$, hence are analytic functions on $U$. For $f=\sum a_{\nu} \xi^{\nu} \in K\langle\xi\rangle$, define the Gauss norm $\|f\|$ of $f$ by

$$
\|f\|:=\max _{\nu}\left|a_{\nu}\right|_{t} .
$$

The ring of overconvergent power series is

$$
K\langle\langle\xi\rangle\rangle:=\bigcup_{\varepsilon>1}\left\{\sum a_{\nu} \xi^{\nu}: \lim _{|\nu| \rightarrow \infty} \varepsilon^{|\nu|}\left|a_{\nu}\right|_{t}=0\right\} .
$$

This is the subring of $K\langle\xi\rangle$ consisting of those power series that converge on some polydisc of $t$-adic radius $>1$. Define the restricted division function $D: K^{2} \rightarrow K^{\circ}$ by

$$
D(x, y):=\left\{\begin{array}{l}
x / y, \quad \text { if }-1 \leq x / y \leq 1 \\
0, \quad \text { else. }
\end{array}\right.
$$

Note that this is defined in terms of the linear order on $K$, rather than in terms of the norm $|\cdot|_{t}$.

Let $\mathcal{L}_{\text {an }}^{D}$ be the language of ordered fields augmented with the function symbol $D$ and function symbols for the overconvergent power series over $K$. The function symbol for the element $f \in K\langle\langle\xi\rangle\rangle$ is interpreted as the function that is zero outside of $[-1,1]^{n}$ and is defined by the power series on $[-1,1]^{n}$. The $t$-adic norm $|\cdot|_{t}$ is not in $\mathcal{L}_{\text {an }}^{D}$.

Definition 1.2. The ring of overconvergent $D$-functions $K\langle\langle\xi, D\rangle\rangle$ is the set of all $\mathcal{L}_{\text {an }}^{D}$-terms in the variables $\xi$ built up from elements of $K\langle\langle\xi, \eta\rangle\rangle$ and $D$ by composition. If $\tau \in K\langle\langle\xi, D\rangle\rangle$ then there are $f \in K\langle\langle\xi, \eta\rangle\rangle, g_{i}, h_{i} \in K\langle\langle\xi, D\rangle\rangle$ such that $\tau=f\left(\xi, D\left(g_{1}, h_{1}\right), \ldots, D\left(g_{m}, h_{m}\right)\right)$.

\section{The ELIMINATiOn THEOREM}

In this section, we prove the main result:

Theorem 2.1. $K$ admits quantifier elimination in $\mathcal{L}_{\text {an }}^{D}$.

Proof. We prove this through a sequence of reductions.

(i) It is sufficient to eliminate quantifiers from formulas of the form

$$
\varphi(\xi)=\varphi_{0}(\xi) \wedge \exists \eta_{1} \cdots \exists \eta_{N}\left[\bigwedge_{i=1}^{L}\left(\tau_{i}(\xi, \eta)=0\right) \wedge \bigwedge_{i=L+1}^{M}\left(0<\tau_{i}(\xi, \eta)\right)\right]
$$

where $\varphi_{0}$ is quantifier-free and the $\tau_{i}$ are $\mathcal{L}_{\text {an }}^{D}$-terms. By standard manipulations, we may assume that all the variables are constrained to lie in the interval $[-1,1]$. 
(ii) At the expense of introducing more $\eta$ 's, we may assume that in the $\tau_{i}$, no variables $\eta$ are within the scope of the function symbol $D$ (recall that the function $D$ is existentially definable.) Hence it is sufficient to eliminate quantifiers from formulas of the form

$$
\varphi(\xi)=\varphi_{0}(\xi) \wedge \exists \eta_{1} \cdots \exists \eta_{N}\left[\bigwedge_{i=1}^{L}\left(f_{i}(\xi, \eta)=0\right) \wedge \bigwedge_{i=L+1}^{M}\left(0<f_{i}(\xi, \eta)\right)\right]
$$

where the $f_{i} \in K\langle\langle\xi, D\rangle\rangle\langle\langle\eta\rangle\rangle$, the subring of $K\langle\langle\xi, \eta, D\rangle\rangle$ consisting of those elements in which $\eta$ does not occur in the scope of any symbol $D$.

(iii) Replacing the terms from $K\langle\langle\xi, D\rangle\rangle$ that occur in the $f_{i}$ by new variables, it is sufficient to eliminate quantifiers from

$$
\begin{aligned}
& \varphi_{0}(\xi) \wedge \bigwedge_{i=1}^{P}\left(\zeta_{i}=D\left(\tau_{i}(\xi), \tau_{i}^{\prime}(\xi)\right)\right) \wedge \\
& \wedge \exists \eta_{1} \cdots \exists \eta_{N}\left[\bigwedge_{i=1}^{L}\left(f_{i}(\zeta, \eta)=0\right) \wedge \bigwedge_{i=L+1}^{M}\left(0<f_{i}(\zeta, \eta)\right)\right],
\end{aligned}
$$

where the $f_{i} \in K\langle\langle\zeta, \eta\rangle\rangle$.

(iv) Let $f_{i}(\zeta, \eta)=\sum_{\nu} a_{i \nu}(\zeta) \eta^{\nu}$, where $a_{i \nu} \in K\langle\langle\zeta\rangle\rangle$. As in [8], Lemma 3.3, using [2], Proposition 5.2.7.1, there is a $d \in \mathbb{N}$ and there are $b_{i \nu \mu} \in K\langle\langle\zeta\rangle\rangle$ for $\mu \in I:=\{0,1, \ldots, d-1\}^{N}$, with $\left\|b_{i \nu \mu}\right\|<1$ and $\left\|b_{i \nu \mu}\right\| \rightarrow 0$ as $|\nu| \rightarrow \infty$, such that

$$
a_{i \nu}=\sum_{\mu \in I} b_{i \nu \mu} a_{i \mu} \text {. }
$$

Hence we can write

$$
f_{i}(\zeta, \eta)=\sum_{\mu \in I} a_{i \mu}(\zeta)\left(\eta^{\mu}+\varphi_{i \mu}(\zeta, \eta)\right)
$$

where the $\varphi_{i \mu} \in K\langle\langle\zeta, \eta\rangle\rangle$ and $\left\|\varphi_{i \mu}\right\|<1$.

(v) Splitting into the cases $a_{i \mu}(\zeta)=0$ and $a_{i \mu}(\zeta) \neq 0$, we can assume that

$$
f_{i}(\zeta, \eta)=\sum_{\mu \in I_{i}} a_{i \mu}(\zeta)\left(\eta^{\mu}+\varphi_{i \mu}(\zeta, \eta)\right)
$$

where $I_{i} \subset\{0, \ldots, d-1\}^{N}$ and for all $\mu \in I_{i}$, the condition on $\zeta$ that $a_{i \mu}(\zeta) \neq 0$ occurs in $\varphi_{0}$.

(vi) We proceed now by induction on $\sum_{i}\left|I_{i}\right|-M$. Assume that $\sum_{i}\left|I_{i}\right|-M=0$ (i.e., $\left|I_{i}\right|=1$, say, $I_{i}=\left\{\mu_{i}\right\}$, for each $i$.) Observe that there is a $\delta<1$ such that for any $\pi \in K$ with $\delta<|\pi|_{t}<1$ we have that

$$
\psi_{i \mu}(\zeta, w):=\varphi_{i \mu}\left(\zeta, \frac{w_{1}}{\pi}, \frac{w_{2}}{\pi^{d}}, \ldots, \frac{w_{N}}{\pi^{d^{N-1}}}\right) \in K\langle\langle\zeta, w\rangle\rangle
$$

and $\left\|\psi_{i \mu}(\zeta, w)\right\|<1$. Choose $\pi=t^{\alpha}, \alpha \in \mathbb{Q}, \alpha>0$, and $|\pi|_{t}$ close enough to 1 , i.e., $\alpha$ close enough to 0 , and make a change of variables $\eta_{N} \mapsto \frac{w_{N}}{\pi}$, and for $i<N$, $\eta_{i} \mapsto w_{i}+\frac{w_{N}}{\pi}$. After multiplying by the constant $\pi^{\alpha\left|\mu_{i}\right|}$, each $\eta^{\mu}+\varphi_{i \mu}(\zeta, \eta)$ becomes regular in $w_{N}$ and we may apply the overconvergent Weierstrass Preparation Theorem (which is an easy consequence of the usual Weierstrass Preparation Theorem) to write

$$
f_{i}=a_{i \mu_{i}}(\zeta) \pi^{\beta} E(w, \zeta) F_{i}(w, \zeta)
$$

where $\beta=-\alpha\left|\mu_{i}\right|, F_{i}(w, \zeta) \in K\left\langle\left\langle\zeta, w_{1}, \ldots, w_{N-1}\right\rangle\right\rangle\left[w_{N}\right]$ and $E(w, \zeta)$ has the property that $\|E-1\|<\|E\|$, so $E$ does not change sign on the closed unit box. Observe that

$$
0 \square f_{i} \leftrightarrow 0 \square a_{i \mu}(\zeta) \pi^{\beta} F_{i}(w, \zeta)
$$


where $\square \in\{=,<\}$, and hence we can apply the classical (Tarski) quantifier elimination to eliminate the quantifier $\exists w_{N}$.

Assume now that for some $I_{i}$ we have $\left|I_{i}\right|>1$. Order the elements of $\{0, \ldots, d-$ $1\}^{N}$ in reverse lexicographic order and let $\mu_{i}$ be the largest element of $I_{i}$. For each such $i$ and $\mu \in I_{i} \backslash\left\{\mu_{i}\right\}$ split into the following cases, defined in terms of the linear order on $K$ :

$$
\begin{aligned}
& \text { Case }(i, *) .\left|a_{i \mu_{i}}\right|_{<} \geq\left|\pi^{\frac{1}{2}} a_{i \mu}\right|_{<} \text {for all } \mu \in I_{i} \backslash\left\{\mu_{i}\right\} . \\
& \text { Case }(i, \mu) .\left|a_{i \mu_{i}}\right|_{<} \leq\left|\pi^{\frac{1}{2}} a_{i \mu}\right|_{<},
\end{aligned}
$$

with $\pi$ chosen as above.

(vii) In the case $(i, \mu)$, we can write

$$
a_{i \mu_{i}}=\pi^{\frac{1}{2}} a_{i \mu} D\left(a_{i \mu_{i}}, \pi^{\frac{1}{2}} a_{i \mu}\right)
$$

and absorb the term $a_{i \mu_{i}}\left(\eta^{\mu}+\varphi_{i \mu_{i}}\right)$ into $\varphi_{i \mu}$, thus decreasing $\sum\left|I_{i}\right|-M$ by 1 . (Replace $D\left(a_{i \mu_{i}}, \pi^{\frac{1}{2}} a_{i \mu}\right)$ by a new $\zeta$-variable.)

(viii) It remains to treat the case $(i, *)$ for all $i$ with $\left|I_{i}\right|>1$. Make the change of variables

$$
\eta_{i}=\pi^{-d^{i-1}} w_{i}
$$

and replace $a_{i \mu}$ by $\pi^{-\frac{1}{2}} a_{i \mu_{i}} D\left(\pi^{\frac{1}{2}} a_{i \mu}, a_{i \mu_{i}}\right)$ for each $\mu \in I_{i} \backslash\left\{\mu_{i}\right\}$. Observe, since $\mu_{i}$ is the largest element of $I_{i}$ with respect to the reverse lexicographic order, that if

$$
a_{i \mu} \eta^{\mu} \mapsto a_{i \mu} \pi^{\beta_{\mu}} w^{\mu}
$$

then $\beta_{\mu_{i}}-1 \geq \beta_{\mu}$ for all $\mu \in I_{i} \backslash\left\{\mu_{i}\right\}$. Hence, after factoring out a suitable power of $\pi$, we can absorb all the terms except $a_{i \mu_{i}} w^{\mu_{i}}$ into $\varphi_{i \mu_{i}}$ and we are in the base case (vi), above.

\section{O-Minimality}

Let $K_{\text {alg }}$ be the algebraic closure of $K$ (so $K_{\text {alg }}=K[\sqrt{-1}]$.) The norm $|\cdot|_{t}$ extends uniquely to $K_{\text {alg }}$. The elements of $K\langle\xi\rangle$ converge on $\left(K_{a l g}^{\circ}\right)^{n}$, and the elements of $K\langle\langle\xi\rangle\rangle$ converge on polydiscs over $K_{\text {alg }}$ of $t$-adic radius greater than 1 .

Definition 3.1. Let $\xi$ be one variable. A $K$-affinoid annulus formula is a formula of the form

$$
\left(\left|P_{0}(\xi)\right|_{t} \leq \varepsilon_{0}\right) \wedge \bigwedge_{i=1}^{N}\left(\varepsilon_{i} \leq\left|P_{i}(\xi)\right|_{t}\right),
$$

where the $\varepsilon_{i} \in\left|K^{\circ} \backslash\{0\}\right|_{t}$ and the $P_{i} \in K^{\circ}[\xi]$ satisfy the following conditions: the $P_{i}$ are monic and irreducible (hence of degree 1 or 2,)

$$
\begin{gathered}
\left\{x \in K_{a l g}:\left|P_{0}(x)\right|_{t} \leq \varepsilon_{0}\right\} \subset K_{\text {alg }}^{\circ}, \\
\left\{x \in K_{\text {alg }}:\left|P_{i}(x)\right|_{t} \leq \varepsilon_{i}\right\} \subset\left\{x \in K_{\text {alg }}:\left|P_{0}(x)\right|_{t} \leq \varepsilon_{0}\right\} \text { for } i=1, \ldots, N, \\
\left\{x \in K_{a l g}:\left|P_{i}(x)\right|_{t}<\varepsilon_{i} \text { and }\left|P_{j}(x)\right|_{t}<\varepsilon_{j}\right\}=\varnothing \text { for } 1 \leq i \neq j \leq N,
\end{gathered}
$$

and if there is an $x \in K$ such that $\left|P_{i}(x)\right|_{t}<\varepsilon_{i}, 1 \leq i \leq N$, then $P_{i}$ is linear, and similarly, if there is an $x \in K$ such that $\left|P_{0}(x)\right|_{t} \leq \varepsilon_{0}$, then $P_{0}$ is linear (i.e., the $P_{i}$ have lowest possible degree.)

A $K$-affinoid annulus is a subset of $K_{\text {alg }}^{\circ}$ that can be defined by a $K$-affinoid annulus formula. 
Note that since there is no symbol for $|\cdot|_{t}$ in $\mathcal{L}_{\text {an }}^{D}$, a $K$-affinoid annulus formula is not an $\mathcal{L}_{\text {an }}^{D}$-formula. The $K$-affinoid annulus formula $\left|\xi^{2}+1\right|_{t} \leq \frac{1}{2}$ is not satisfied by any $K$-rational point; i.e., over $K$, it defines the empty set. Indeed, since by Theorem 3.16, below, $K$ is o-minimal in $\mathcal{L}_{\text {an }}^{D}$, the only $\mathcal{L}_{\text {an }}^{D}$-definable subset of $K$ that can be defined by a $K$-affinoid annulus formula is the empty set.

Remark 3.2. If the polynomial $P_{0}$ of Definition 3.1 is linear, then the subset $U$ of $K_{\text {alg }}^{\circ}$ defined by the $K$-affinoid annulus formula of Definition 3.1 is a standard set, as in [2], Section 9.7.2; otherwise, it is the disjoint union of two conjugate standard sets. In either case, it is clear from Definition 3.1 that $U$ is a rational subdomain of $K_{\text {alg }}^{\circ}$ (for the definition of rational domain, see [2], Section 7.2.3.) Therefore, a $K$-affinoid algebra $\mathcal{O}_{K}(U)$ is canonically associated to $U$, and

$$
\begin{gathered}
\mathcal{O}_{K}(U) \simeq K\left\langle\xi, \eta_{0}, \ldots, \eta_{N}\right\rangle /\left(P_{0}-\alpha_{0} \eta_{0}, \eta_{1} P_{1}-\alpha_{1}, \ldots, \eta_{N} P_{N}-\alpha_{N}\right)= \\
=K\left\langle\xi, \frac{P_{0}}{\alpha_{0}}, \frac{\alpha_{1}}{P_{1}}, \ldots, \frac{\alpha_{N}}{P_{N}}\right\rangle
\end{gathered}
$$

where the $\alpha_{i} \in K$ satisfy $\left|\alpha_{i}\right|_{t}=\varepsilon_{i}$. Alternatively, $\mathcal{O}_{K}(U)$ is the completion of the localization $K[\xi]_{P}$ (where $P=P_{1} \cdots P_{N}$ ) with respect to the supremum norm over $U$ (see [6], Section 2.2.) The $K_{a l g}$-affinoid algebra canonically associated to $U, \mathcal{O}_{K_{a l g}}(U)$, is defined similarly. Since $K_{a l g}$ is finite over $K$,

$$
\mathcal{O}_{K_{\text {alg }}}(U) \simeq \mathcal{O}_{K}(U) \otimes_{K} K_{\text {alg }}
$$

The complex conjugation $\sigma$ of $K_{\text {alg }}$ over $K$ extends to an $\mathcal{O}_{K}(U)$-algebra automorphism of $\mathcal{O}_{K_{\text {alg }}}(U)$. As mentioned above, there are two possibilities, either $U$ is a standard subset of $K_{a l g}^{\circ}$, or it is the disjoint union of two standard subsets of $K_{\text {alg }}^{\circ}, U_{1}$ and $U_{2}$, which are the Zariski-irreducible components of $U$ with respect to $\mathcal{O}_{K_{\text {alg }}}(U)$. In the latter case, by [6], Lemma 2.2.8 (see also [2], Proposition 7.2.2.9,)

$$
\mathcal{O}_{K_{\text {alg }}}(U)=\mathcal{O}_{K_{\text {alg }}}\left(U_{1}\right) \oplus \mathcal{O}_{K_{\text {alg }}}\left(U_{2}\right) \text {, }
$$

and $\sigma$ is an $\mathcal{O}_{K}(U)$-algebra isomorphism of $\mathcal{O}_{K_{\text {alg }}}\left(U_{1}\right)$ with $\mathcal{O}_{K_{\text {alg }}}\left(U_{2}\right)$.

Lemma 3.3. Let $f \in K(\xi)$ be a rational function. Then the set

$$
\left\{x \in K^{\circ}:|f(x)|_{t} \leq 1\right\}
$$

is a finite union of $K$-affinoid annuli.

Proof. This is a small extension of [6], Exercises 2.1.1 and 2.1.2. See also [3], Lemma 3.16.

Definition 3.4. Let $U$ be a $K$-affinoid annulus. An element $f \in \mathcal{O}_{K_{\text {alg }}}(U)$ is called a strong unit if, and only if, there is a $c \in K_{\text {alg }} \backslash\{0\}$ such that (i) $|c \cdot f(x)|_{t}=1$ for all $x \in U$, and (ii) the set of residues modulo $K_{\text {alg }}^{\circ \circ}$ of $c \cdot f(x), x \in U$, is finite.

Remark 3.5. (i) Note that if $f \in \mathcal{O}_{K_{a l g}}(U)$ is a strong unit, then so is $f^{-1}$, and the product of strong units is a strong unit.

(ii) The simplest example of a strong unit is a non-zero constant.

(iii) Consider the unit disc $U:=K_{\text {alg }}^{\circ}$, and let $a \in K_{\text {alg }} \backslash K_{\text {alg }}^{\circ}$; then $f:=(a-\xi)$ is a strong unit of $\mathcal{O}_{K_{\text {alg }}}(U)$ because $\left|a^{-1} \cdot f(x)\right|_{t}=1$ for all $x \in U$ by the ultrametric inequality, and the set of residues modulo $K_{\text {alg }}^{\circ \circ}$ of $a^{-1} \cdot f(x)$ is $\{1\}$.

(iv) Consider the $K$-affinoid annulus $U:=\left\{x \in K_{\text {alg }}:\left|x^{2}+1\right|_{t} \leq|t|_{t}\right\}$. Then $U$ has two $K_{a l g}$-components, namely, the discs of radius $|t|_{t}$ centered at $\pm \sqrt{-1}$. 
Observe that $f:=\xi$ is a strong unit in $\mathcal{O}_{K}(U)$ because $|f(x)|_{t}=1$ for all $x \in U$ and the set of residues modulo $K_{\text {alg }}^{\circ \circ}$ of $f(x)$ is $\{ \pm \sqrt{-1}\}$. Note that in $\mathcal{O}_{K}(U)$,

$$
f^{-1}=-\xi \cdot \sum_{n=0}^{\infty}\left(1+\xi^{2}\right)^{n}
$$

and $\left|1+x^{2}\right|_{t} \leq|t|_{t}$ for all $x \in U$, so the infinite sum is an element of $\mathcal{O}_{K}(U)$.

(v) Suppose that $U$ is a $K$-affinoid annulus with only one $K_{\text {alg-component; equiv- }}$ alently, $\sigma(U)=U$, or $U \cap K \neq \varnothing$. Suppose that $f \in \mathcal{O}_{K}(U)$ is a strong unit and fix $c \in K \backslash\{0\}$ such that $|c \cdot f(x)|_{t}=1$ for all $x \in U$. Then there is a fixed $a \in K^{\circ}$ such that for all $x \in U$ the residue of $c \cdot f(x)$ equals the residue of $a$ modulo $K_{\text {alg }}^{\circ \circ}$. Since $f=\sigma(f)$, this follows from the proof of [6], Theorem 2.2.9 (see the proof of Lemma 3.6, below.)

Lemma 3.6. Let $U$ be a $K$-affinoid annulus defined by the $K$-annulus formula

$$
\left(\left|P_{0}(\xi)\right|_{t} \leq \varepsilon_{0}\right) \wedge \bigwedge_{i=1}^{N}\left(\varepsilon_{i} \leq\left|P_{i}(\xi)\right|_{t}\right)
$$

Then each element $f \in \mathcal{O}(U)$ uniquely determines the following data: a strong unit $E$ of $\mathcal{O}(U)$, a monic polynomial $g \in K[\xi]$, all the zeros of which lie in $U$, and non-negative integers $n_{i}$ such that

$$
f=E \cdot \frac{g}{\prod_{i=1}^{N} P_{i}^{n_{i}}} .
$$

Proof. The corresponding result over $K_{\text {alg }}$ for each irreducible component of $U$ is in the proof of [6], Theorem 2.2.9. Let $f \in \mathcal{O}_{K}(U)$. In case $U$ is irreducible (i.e., $P_{0}$ is linear) we have

$$
f=E \cdot R,
$$

where $E$ is a strong unit of $\mathcal{O}_{K_{a l g}}(U)$ and $R \in K_{a l g}(\xi)$ is a rational function of the appropriate form. Since $\sigma(f)=f$, by the uniqueness of such expressions, $\sigma(R)=R$, so $R \in K(\xi)$ and $E$ is a strong unit of $\mathcal{O}_{K}(U)$.

In the remaining case, $U$ is a disjoint union of the standard sets $U_{1}$ and $U_{2}$, and for $i=1,2$, we have decompositions

$$
\left.f\right|_{U_{i}}=E_{i} \cdot R_{i}
$$

with respect to $\mathcal{O}_{K_{\text {alg }}}\left(U_{i}\right)$, and $\sigma\left(E_{1}\right)=E_{2}, \sigma\left(R_{1}\right)=R_{2}$. Put $R:=R_{1} \cdot R_{2} \in K(\xi)$. We have

$$
\left.f\right|_{U_{1}}=\left(E_{1} R_{2}^{-1}\right) R
$$

Since $R \in K(\xi), \sigma\left(E_{1} R_{2}^{-1}\right)=E_{1} R_{2}^{-1}$, so $E:=E_{1} R_{2}^{-1} \in \mathcal{O}_{K}(U)$. Since $R$ is of the required form, it remains to see that $E$ is a strong unit. But by Remark 3.5, $R_{2}$ is a strong unit of $\mathcal{O}_{K_{a l g}}\left(U_{1}\right)$, so $E \in \mathcal{O}_{K}(U)$ is a strong unit of $\mathcal{O}_{K_{a l g}}\left(U_{1}\right)$, and since $\sigma(E)=E, E$ must be a strong unit of $\mathcal{O}_{K_{a l g}}\left(U_{2}\right)$. Thus $E$ is a strong unit of $\mathcal{O}_{K}(U)$, as desired. The uniqueness of the expression $f=E \cdot R$ follows from the uniqueness for the $\mathcal{O}_{K_{a l g}}\left(U_{i}\right)$.

Corollary 3.7. Suppose that $U$ is a $K$-affinoid annulus and that $U \cap K \neq \varnothing$. Let $f \in \mathcal{O}_{K}(U)$ be a strong unit; then $f$ has constant sign on $U \cap K$. 
Proof. By Remark $3.5(\mathrm{v})$, there are $c \in K \backslash\{0\}$ and $a \in K^{\circ} \backslash K^{\circ \circ}$ such that for all $x \in U$,

$$
\left|f(x)-c^{-1} a\right|_{t}<\left|c^{-1} a\right|_{t} .
$$

It follows that the sign of $f(x)$ coincides with the sign of $c^{-1} a$ for all $x \in U$.

Definition 3.8. Let $U$ be a $K$-affinoid annulus. A subset $X$ of $U \cap K$ is called relatively semialgebraic in $U$ if, and only if, there is a semialgebraic subset $Y$ of $K$ (which, by Tarski's theorem, is a finite union of intervals with endpoints in $K \cup\{ \pm \infty\})$ such that $X=Y \cap(U \cap K)$.

Note that if $X$ is relatively semialgebraic in $U$, then so is $U \backslash X$.

Lemma 3.9. Let $U$ be a $K$-affinoid annulus such that $U \cap K \neq \varnothing$, let $f, g \in \mathcal{O}_{K}(U)$. Then: (i) the set

$$
\left\{x \in U:|f(x)|_{<}=|g(x)|_{<}\right\}
$$

is either finite or equal to $U$, and (ii) the set

$$
\left\{x \in U:|f(x)|_{<} \leq|g(x)|_{<}\right\}
$$

is relatively semialgebraic in $U$.

Proof. Taking $h:=g^{2}-f^{2}$, it is sufficient to consider the sets

$$
\begin{aligned}
& X:=\{x \in U: 0=h(x)\} \\
& Y:=\{x \in U: 0 \leq h(x)\}
\end{aligned}
$$

By Lemma 3.6 and Corollary 3.7, we may assume that $h \in K(\xi)$ is a rational function with no poles in $U$.

Lemma 3.10. Let $f \in K\langle\langle\xi, D\rangle\rangle$. Then there is a finite cover $\mathcal{U}$ of $K_{\text {alg }}^{\circ}$ by $K$ affinoid annuli, a finite cover $\mathcal{X}_{U}$ of each set $U \cap K, U \in \mathcal{U}$, by sets relatively semialgebraic in $U \cap K$, and for each $U \in \mathcal{U}$ and $X \in \mathcal{X}_{U}$, there is an element $f_{U X} \in \mathcal{O}_{K}(U)$ such that

$$
\left.f\right|_{(X \cap U)}=\left.f_{U X}\right|_{(X \cap U)} .
$$

Proof. We may write

$$
f=F\left(\xi, D\left(f_{1}, g_{1}\right), \ldots, D\left(f_{n}, g_{n}\right)\right),
$$

where $F \in K\left\langle\left\langle\eta_{0}, \ldots, \eta_{n}\right\rangle\right\rangle$ and the $f_{i j} \in K\langle\langle\xi, D\rangle\rangle$. We proceed by induction on the number of occurrences of $D$, at each stage producing a refinement of the pair of covers produced at the previous stage. Note that the intersection of two $K$ affinoid annuli is either a $K$-affinoid annulus or the empty set, and the intersection of two semialgebraic sets is semialgebraic. Since $F$ is overconvergent, there is some $\varepsilon \in|K \backslash\{0\}|_{t}, \varepsilon>1$, such that $F$ converges on the polydisc of radius $\varepsilon$. For $i=1, \ldots, n$, suppose we have covers $\mathcal{U}_{i}$ and $\left\{\mathcal{X}_{i U}\right\}_{U \in \mathcal{U}_{i}}$, as in the lemma, such that for each $U \in \mathcal{U}_{i}$ and $X \in \mathcal{X}_{i U}$ there is an $h_{i U X} \in \mathcal{O}_{K}(U)$ such that

$$
\left.D\left(f_{i}, g_{i}\right)\right|_{(X \cap U)}=\left.h_{i U X}\right|_{(X \cap U)}
$$

and

$$
\left\|h_{i U X}\right\|_{\mathrm{sup}, U} \leq \varepsilon
$$

where $\|\cdot\|_{\text {sup }, U}$ is the supremum norm on the affinoid domain $U$ (as in [2], Section 6.2.1.) Then, taking a common refinement of these covers and the corresponding compositions with $F$ establishes the lemma. Hence it suffices to prove the following: 
Let $W \subset K_{\text {alg }}^{\circ}$ be a $K$-affinoid annulus, let $Y$ be a relatively semialgebraic subset of $W \cap K$ and let $f, g \in \mathcal{O}_{K}(W)$; then there is a finite cover $\mathcal{U}$ of $W$ by $K$-affinoid annuli, a finite cover $\mathcal{X}_{U}$ of each set $U \cap Y, U \in \mathcal{U}$, by relatively semialgebraic sets, and for each $U \in \mathcal{U}$ and $X \in \mathcal{X}_{U}$, there is an element $h_{U X} \in \mathcal{O}_{K}(U)$ such that

$$
\left.D(f, g)\right|_{(X \cap U \cap Y)}=\left.h_{U X}\right|_{(X \cap U \cap Y)}
$$

and

$$
\left\|h_{U X}\right\|_{\sup , U} \leq \varepsilon
$$

By Lemma 3.6, $\mathcal{O}_{K}(W)$ is a principal ideal domain, so by the Nullstellensatz (see [2], Proposition 7.1.3.1,) for $h \in \mathcal{O}_{K}(W)$, if $h(a)=0$, then there is an $h^{\prime} \in \mathcal{O}_{K}(W)$ such that $h=(\xi-a) h^{\prime}$. We are only concerned with the values of the ratio $f / g$, except possibly at the finitely many points of the set $Z$ of common "real" (i.e., $K$ rational) zeros of the pair $f$ and $g$, which we can handle separately. Hence, using Lemma 3.6 and the Nullstellensatz to factor out any common factors and replacing $Y$ by $Y \backslash Z$, we may assume that $f$ and $g$ have no common zero on $W$.

By Lemma 3.6 and Lemma 3.3, the sets

$$
\begin{aligned}
& A:=\left\{x \in W:|f(x)|_{t} \leq \varepsilon|g(x)|_{t}\right\} \text { and } \\
& B:=\left\{x \in W:|f(x)|_{t} \geq \varepsilon|g(x)|_{t}\right\}
\end{aligned}
$$

are each finite unions of $K$-affinoid annuli. Since $W=A \cup B$, intersecting with these $K$-affinoid annuli, we can reduce to the cases that $W \subset A$ or $W \subset B$.

Case 1. $W \subset A$.

Since $f$ and $g$ have no common zero on $W$, by the condition defining $A, g$ is a unit in $\mathcal{O}_{K}(W)$, so $f / g \in \mathcal{O}_{K}(W)$ and $\|f / g\|_{\text {sup }, W} \leq \varepsilon$. Put

$$
\begin{aligned}
& X_{1}:=Y \cap\left\{x \in W:|f(x)|_{<} \leq|g(x)|_{<}\right\} \\
& X_{2}:=Y \cap\left\{x \in W:|f(x)|_{<}>|g(x)|_{<}\right\} ;
\end{aligned}
$$

then by Lemma 3.9, $X_{1}$ and $X_{2}$ are relatively semialgebraic in $W$. Put

$$
h_{W X_{1}}:=f / g \text { and } h_{W X_{2}}:=0
$$

and note that

$$
\left.D(f, g)\right|_{X_{1}}=\left.h_{W X_{1}}\right|_{X_{1}} \quad \text { and }\left.\quad D(f, g)\right|_{X_{2}}=\left.h_{W X_{2}}\right|_{X_{2}} .
$$

Case 2. $W \subset B$.

By the condition defining $B$, for all $x \in W$,

$$
|f(x)|_{<}>|g(x)|_{<} .
$$

Put

$$
h_{W Y}:=0
$$

then

$$
\left.D(f, g)\right|_{(Y \cap W)}=\left.h_{W Y}\right|_{(Y \cap W)} .
$$

The following technical lemmas are needed for the proof of Theorem 3.16.

Lemma 3.12. Let $U_{1}, \ldots, U_{n} \subset K_{\text {alg }}^{\circ}$ be $K$-affinoid annuli that cover $K^{\circ \circ}$. Then there are real numbers $a$ and $b,-1 \leq a<0<b \leq 1$, such that $U_{1}, \ldots, U_{n}$ cover the closed interval $[a, b]$. 
Proof. Let $C \subset K^{\circ}$ be a collection of "centers," one for each "hole" that meets $K$ in each of the $U_{i}$. Let $a \in \mathbb{R},-1 \leq a<0$, satisfy $c<a$ for all $c \in C$ with $|c|_{t}=1$ and $c<0$, and let $b \in \mathbb{R}, 0<b \leq 1$, satisfy $b<c$ for all $c \in C$ with $|c|_{t}=1$ and $c>0$.

Lemma 3.13. Let $U_{1}, \ldots, U_{n} \subset K_{\text {alg }}^{\circ}$ be $K$-affinoid annuli that cover $[a, b], a, b \in$ $K$. Then there is a finite collection $\mathcal{X}$ of closed intervals with endpoints in $K$ such that $\mathcal{X}$ covers $[a, b]$ and $\mathcal{X}$ refines $\left\{U_{i} \cap[a, b]\right\}_{1 \leq i \leq n}$.

Proof. Induct on $n$. If $n=1$, there is nothing to prove. At least one of the $U_{i}$ must have radius at least $|b-a|_{t}$, so by renumbering, we may assume that the "outer disc" of $U_{n}$ contains $[a, b]$. Let $V_{1}^{\circ \circ}, \ldots, V_{m}^{\circ \circ}$ be the (open) "holes" of $U_{n}$ and let $V_{1}^{\circ}, \ldots, V_{m}^{\circ}$ be the corresponding closed discs. Each $V_{j}^{\circ \circ}$ is covered by $U_{1}, \ldots, U_{n-1}$, so by translating and rescaling and Lemma 3.12 , there are $a_{j}, b_{j} \in$ $\left(K \cap V_{j}^{\circ}\right) \backslash V_{j}^{\circ \circ}$ such that $V_{j}^{\circ \circ} \cap K \subset\left[a_{j}, b_{j}\right]$ and $U_{1}, \ldots, U_{n-1}$ cover $\left[a_{j}, b_{j}\right]$. Note that $[a, b] \backslash \bigcup_{j=1}^{m}\left(a_{j}, b_{j}\right)$ is the union of a finite collection of closed intervals with endpoints in $K$, all contained in $U_{n}$. Hence, by induction, we are done.

Lemma 3.14. Let $\mathcal{U}$ be a finite cover of $[-1,1]$ by $K$-affinoid annuli. For each $U \in \mathcal{U}$, suppose that $X_{U} \subset(U \cap K)$ is a relatively semialgebraic set. Suppose for each $U, V \in \mathcal{U}$ that

$$
X_{U} \cap V=X_{V} \cap U .
$$

Then $[-1,1] \cap \bigcup_{U \in \mathcal{U}} X_{U}$ is semialgebraic.

Proof. By Lemma 3.13, there are elements $a_{0}, \ldots, a_{n} \in K$ with $-1=a_{0}<a_{1}<$ $\cdots<a_{n}=1$ such that for each $i=0, \ldots, n-1$, there is some $U_{i} \in \mathcal{U}$ such that $\left[a_{i}, a_{i+1}\right] \subset U_{i}$. Note that $X_{U_{i}} \cap\left[a_{i}, a_{i+1}\right]$ is semialgebraic and condition (3.15) implies that

$$
[-1,1] \cap \bigcup_{U \in \mathcal{U}} X_{U}=\bigcup_{i}\left(\left[a_{i}, a_{i+1}\right] \cap X_{U_{i}}\right)
$$

Theorem 3.16. The structure $K$ is o-minimal in $\mathcal{L}_{\text {an }}^{D}$.

Proof. Let $X \subset K$ be $\mathcal{L}_{\text {an }}^{D}$-definable. It is sufficient to consider the case $X \subset$ $[-1,1]$. By the quantifier elimination theorem, Theorem 2.1, $X$ is quantifier-free $\mathcal{L}_{\text {an }}^{D}$-definable. Thus by Lemma 3.10 and Lemma 3.9, there is a finite cover $\mathcal{U}$ of $[-1,1]$ by $K$-affinoid annuli, and for each $U \in \mathcal{U}$, the set $X_{U}:=U \cap X$ is relatively semialgebraic. Therefore, by Lemma $3.14, X$ is semialgebraic.

Fix $a \in K$. Note that if one replaces, in the definition of $\mathcal{L}_{\text {an }}^{D}$, the rings of power series convergent in polydiscs of radius greater than 1 by the rings of power series convergent in polydiscs of radius greater than $|a|_{t}$, one obtains exactly the same class of definable sets. Indeed, if $f(\xi)$ converges on a polydisc of radius greater than $|a|_{t}$ then $g(\eta):=f(a \cdot \eta) \in K\langle\langle\eta\rangle\rangle$ and for all $x, f(x)=g(D(x, a))$. In particular, if $a=t$, then each element of $\mathbb{R} \llbracket \xi \rrbracket$ converges in $|\cdot|_{t}$ on a polydisc of radius greater than $|t|_{t}$ (in fact, $f$ converges on all of $\left(K^{\circ \circ}\right)^{n}$.) Hence $f$ defines a function $[-t, t]^{n} \rightarrow K^{\circ}$. The following is thus an immediate corollary of Theorem 3.16.

Corollary 3.17. Let $\mathcal{L}$ be the language of ordered fields with function symbols for the elements of $\bigcup_{n} \mathbb{R} \llbracket \xi_{1}, \ldots, \xi_{n} \rrbracket$, interpreted as analytic functions on $[-t, t]^{n}$; then $K$ is o-minimal in the language $\mathcal{L}$. 


\section{REFERENCES}

[1] A. Berarducci and T. Servi, "An effective version of Wilkie's theorem of the complement and some effective o-minimality results," Ann. Pure Appl. Logic 125 (2004) 43-74.

[2] S. Bosch, U. Güntzer and R. Remmert, Non-archimedean Analysis, Springer-Verlag (1984).

[3] R. Cluckers, L. Lipshitz and Z. Robinson, "Analytic cell decomposition and analytic motivic integration," 32 pp., submitted 2005 (available at http://front.math.ucdavis.edu/math.AG/0503722).

[4] J. Denef, L. van den Dries, "p-adic and real subanalytic sets," Ann. Math. 128 (1988) 79-138.

[5] L. van den Dries, "o-minimal structures," in: Logic: from Foundations to Applications, Clarendon Press, Oxford (1996), pp 137-185.

[6] J. Fresnel and M. van der Put, Rigid Analytic Geometry and Its Applications, Birkhäuser (2004).

[7] E. Hrushovski and Y. Peterzil, "A question of van den Dries and a theorem of Lipshitz and Robinson; not everything is standard," preprint (available at http://math.haifa.ac.il/kobi/Lou-question-2.ps).

[8] H. Schoutens, "Rigid subanalytic sets," Compositio Math. 94 (1994), 269-295.

Department of Mathematics, Purdue University, 150 North University Street, West LAFAYETTE IN 47907-2067 USA

E-mail address: lipshitz@math.purdue.edu

Department of Mathematics, East Carolina University, Greenville NC 27858 USA

E-mail address: robinsonz@mail.ecu.edu 